\title{
FUNCIONES COGNITIVAS EN ESTUDIANTES QUE INGRESAN A EDUCACIÓN SUPERIOR1
}

Cognitive functions of students entering higher education

Funções cognitivas em estudantes que ingressam na Educação Superior

\author{
Ana Maria Vásquez Aqueveque \\ Universidad de La Serena \\ amvas@userena.cl \\ Silvia López de Maturana Luna \\ Universidad de La Serena \\ silvialml@gmail.com \\ Ken Isamu Matsuda Oteiza (†) \\ Universidad de La Serena \\ kmatsuda@userena.cl \\ Pamela Labra Godoy \\ Universidad de La Serena \\ 1labra@userena.cl

\section{Resumen}

Estudio exploratorio y descriptivo, sustentado en la teoría de la Modificabilidad Cognitiva de Reuven Feuerstein (1991), sobre la autopercepción de las funciones cognitivas (FC) de los(as) estudiantes que ingresan a la Universidad de La Serena, Chile.2 Su desarrollo es

\footnotetext{
1 Este trabajo se enmarca en el Concurso de Propuestas de Planes de Mejoramiento de Programas (PM) del Ministerio de Educación de Chile, denominado "Fortalecimiento de la Unidad de Mejoramiento Docente de la Universidad de La Serena, mediante la creación e implementación del área de Innovación y Transferencia en aprendizaje, docencia y gestión curricular: una mejora sistémica en el proceso de Innovación Curricular".

2 Estudiantes de las carreras de Auditoría, Derecho, Enfermería, Ingeniería en Administración de Empresa, Ingeniería Comercial, Ingeniería en Alimentos, Kinesiología, Licenciatura en Astronomía y Odontología.
} 
crucial para la complejidad del aprendizaje universitario. Se seleccionó una muestra de 112 alumnos que respondieron a una pauta de FC en las tres fases del acto mental: entrada, elaboración, salida. La confiabilidad se calculó por el alpha de Cronbach (0.954) y la validez de constructo se determinó por medio de un análisis factorial confirmatorio. Los hallazgos revelan que independientemente de la carrera a la que ingresan los estudiantes y del año de incorporación a la Educación Superior, hay similitud en la autopercepción de los/as estudiantes sobre el descenso de algunas FC, por ejemplo, precisión y exactitud, atención a dos o más fuentes de información, amplitud del campo mental, transporte visual y desinhibición de la respuesta, las que han sido provocadas por la enseñanza escolarizada. Activar el desarrollo de esas funciones, relevantes para la adquisición de aprendizajes profundos, demanda de sus profesores/as experiencias de aprendizaje mediado (EAM) para que los estudiantes se modifiquen cognitivamente.

Palabras clave: funciones cognitivas; aprendizaje profundo; educación superior; mediación pedagógica; acto mental.

\begin{abstract}
Exploratory and descriptive study, based on the theory of Cognitive Modifiability of Reuven Feuerstein (1991), on the self-perception of cognitive functions (CF) and its development in students entering the University of La Serena, Chile, given that their development it is crucial to the complexity of university learning. A sample of 112 students who responded to a CF pattern in the three phases of the mental act: entrance, elaboration, and exit. Reliability was calculated by Cronbach's alpha (0.954) and construct validity was determined by confirmatory factor analysis. The findings reveal that regardless of the program to which students enter and the year of entry into Higher Education, there is similarity in their self-perception regarding the decrease of some CF; for example, accuracy and precision, attention to two or more sources of information, width of the mental field, visual transport and disinhibition of the response, which have been caused by schooling. Activating the development of these functions is relevant to the acquisition of deep learning. It requieres from their professors mediated learning experiences (MLE) so as to cognitively modify themselves.
\end{abstract}


Key words: Cognitive functions; deep learning; higher education; pedagogical mediation; mental act.

\section{Resumo}

Estudo exploratório e descritivo, sustentado na teoría da Modificabilidade Cognitiva de Reuven Feuerstein (1991), sobre a autopercepção das funções cognitivas(FC) dos(as) estudantes que ingressam na Universidade de La Serena, Chile3. Seu desenvolvimento é crucial para a complexidade da aprendizagem universitária. Selecionou-se uma amostra de 112 alunos que responderam a uma pauta de FC nas três fases do ato mental: entrada, elaboração, saída. A confiabilidade foi calculada pelo alpha de Cronbach(0.954) e a validade do construto foi determinada por meio de uma análise fatorial confirmatória. As descobertas revelam que independentemente da carreira na qual ingressam os estudantes e do ano de incorporação à Educação Superior, há similitude na autopercepção dos estudantes sobre o descenso de algunas FC, por exemplo, precisão e exatidão; atenção a duas ou mais fontes de informação; amplitude do campo mental; transporte visual e desinibição da resposta, consequências provocadas pelo ensino escolarizado. Ativar o desenvolvimento dessas funções, relevantes à aquisição de aprendizagens profundas, demanda de seus professores(as) experiências de aprendizagem mediada (EAM) para que os estudantes se modifiquem cognitivamente.

Palavras-chave: funções cognitivas; aprendizagem profunda; educação superior; mediação pedagógica; ato mental.

\section{Introducción}

Hemos indagado la autopercepción de las funciones cognitivas (FC) que tienen estudiantes de primer año de nueve carreras4 que han renovado los planes de estudio en la Universidad de La Serena (ULS)5. Hemos observado que muchos/as tienen problemas de aprendizaje

3 Estudiantes dos cursos de Auditoria, Direito, Enfermagem, Engenharia em Administração de empresas, Engenharia Comercial, Engenharia de Alimentos, Cinesiologia, Licenciatura em Astronomia e Odontologia.

4 Carreras de Auditoría, Derecho, Enfermería, Ingeniería en Administración de Empresa, Ingeniería Comercial, Kinesiología, Licenciatura en Astronomía y Odontología.

5 El Modelo Educativo (2011) declarado por la institución, concibe al estudiante como el centro de su quehacer formativo. 
porque no aprovechan su potencial cognitivo. Aprenden superficialmente y sus profesores/as no reparan en ello. La consecuencia directa es que fracasan. Algunos se conforman con la ilusión de haber aprendido y algunos profesores/as de haber simplemente enseñado (Morin, 2001). Esto favorece el desarrollo de enfermedades paidogénicas, es decir, causadas por el mismo profesorado (Ferguson 1988). De este modo, muchos/as estudiantes predicen su "profecía autocumplida" (García Pintrich, 1994; Midgley et al. 1996, citado en Pintrich, 2004) cuando asumen que no pueden aprender más que lo mínimo al autoimponerse obstáculos, generando resistencias, disminuyendo el esfuerzo, estudiando para la prueba, etc..

El interés por indagar las FC de los/as estudiantes radica en que las dificultades de aprendizaje que manifiestan, en gran medida, son la consecuencia de disfunciones cognitivas que se consolidaron durante los primeros años de vida e inhibieron su desarrollo normal. Las FC son estructuras dinámicas esenciales para el funcionamiento de la mente. Forman parte de una complejidad de componentes interdependientes que se expresan en patrones de conductas distinguibles y necesarias para detectar las causas de estas dificultades (Feuerstein, 1991). Esto permite observarlas a través del comportamiento de los/as estudiantes y/o a través de su autopercepción, como es este caso.

Consideramos que si el profesorado asume su papel de mediador y no solo de expositor de contenidos ayudaría a superar las disfunciones cognitivas de sus estudiantes y evitaría los daños en el comportamiento académico, la autoestima y el autoconcepto del estudiantado (Tébar, 2007). De este modo, recupereremos el rol de la educación y descartaremos el de la escolarización6.

El educador mediador, no escolarizado, considera que el/la estudiante constituye el centro del proceso de enseñanza y de aprendizaje y que él orienta los procesos. Comprende que enseñar y aprender son dos procesos diferentes que no tienen entre sí relación de causalidad lineal unívoca sino de mutua complementariedad. Desafortunadamente, es muy frecuente

6 Distinguimos entre educación y escolarización, entendiendo la primera como la creación de relaciones posibles y la segunda como la repetición de relaciones preestablecidas (Calvo, 2016). Ambos procesos pueden darse en la escuela. 
que muchos/as docentes asuman que basta enseñar para asegurar el aprendizaje. De acuerdo a Feuerstein (1991), el profesorado mediador crea una EAM7 cuando enseña de manera sencilla y directa atendiendo al desarrollo cognitivos de sus estudiantes; les ayuda a superar las dificultades que les impiden comprender; también selecciona estrategias de enseñanza, procedimientos, instrumentos de evaluación, etc. En la investigación identificaremos que saben los/as estudiantes sobre sus procesos de aprendizaje y como usan sus FC. Gracias a ello, el profesorado podrá mejorar la enseñanza y los/as estudiantes el aprendizaje.

Las FC se organizan en las tres fases del Acto Mental: Entrada, Elaboración y Salida de la información (Feuerstein 1991). A saber:

Tabla 1: Funciones cognitivas en las tres fases del acto mental

\begin{tabular}{|c|c|c|}
\hline $\begin{array}{c}\text { Fase de entrada de la } \\
\text { información }\end{array}$ & $\begin{array}{c}\text { Fase de elaboración de la } \\
\text { información }\end{array}$ & Fase de salida de la información \\
\hline Percepción clara & $\begin{array}{l}\text { Percepción y definición de un } \\
\text { problema }\end{array}$ & Comunicación explícita \\
\hline $\begin{array}{l}\text { Exploración sistemática de una } \\
\text { situación de aprendizaje }\end{array}$ & Distinción de datos relevantes & Proyección de relaciones virtuales \\
\hline Habilidades lingüísticas & Conducta comparativa & $\begin{array}{l}\text { Restricción de las respuestas por } \\
\text { ensayo y error }\end{array}$ \\
\hline Orientación espacial & Amplitud del campo mental & Habilidades lingüísticas \\
\hline Orientación temporal & $\begin{array}{l}\text { Organización y estructuración } \\
\text { perceptiva }\end{array}$ & Transporte visual \\
\hline $\begin{array}{l}\text { Constancia y permanencia del } \\
\text { objeto }\end{array}$ & Evidencia lógica & Precisión y exactitud \\
\hline Precisión y exactitud & $\begin{array}{l}\text { Interiorización y representación } \\
\text { mental }\end{array}$ & Control de la respuesta \\
\hline \multirow[t]{2}{*}{$\begin{array}{l}\text { Atención a dos o más fuentes de } \\
\text { información }\end{array}$} & Pensamiento hipotético inferencial & $\begin{array}{l}\text { Elaboración y desinhibición en la } \\
\text { comunicación }\end{array}$ \\
\hline & Planificación de la conducta & \\
\hline
\end{tabular}

Fuente: Basada en Feuerstein (1991), Tebar (2007), Martinez (1991), Prieto (1989)

Si los/as estudiantes no han desarrollado las FC de la fase de Entrada de la información probablemente tendrían dificultades para explorar sistemáticamente un problema, atender a dos o más fuentes de información, comprender el vocabulario, tomar los datos con precisión, etc. Si existen FC deficientes en la fase de Elaboración tendrán dificultades para identificar un problema, plantear hipótesis, comparar, interiorizar, identificar datos

7 Feuerstein (1991) describe a la Experiencia de Aprendizaje Mediado como la interrelación afectiva y efectiva del mediador entre los estímulos y el sujeto mediado y entre éste y su respuesta, para que el proceso de aprendizaje resulte significativo y trascendente. 
relevantes, etc. Si existen FC deficientes en la fase de Salida tendrán dificultades para entregar una respuesta precisa, utilizar las palabras adecuadas para responder de manera fluida y coherente, y para proyectar relaciones virtuales, etc. Al tomar consciencia de las ventajas y desventajas del desarrollo cognitivo, los/as estudiantes consolidan aprendizajes profundos y evitan la vaguedad y superficialidad (Entwistle, 1981, Ramsdem, 1992, y Biggs, 1993 citados por Facse, 2007).

A medida que el/la estudiante va desarrollando sus FC siente más seguridad en si mismo/a, se anima a relacionar y a preguntar sin temor; aumenta su autoconfianza y disminuye su ingenuidad; regula su conducta y organiza el tiempo de mejor manera. De todas maneras, el profesorado debe estar atento a esos cambios, pues si provoca conflictos cognitivos dificultades que el/la estudiante no podrá resolver inmediatamente, sino que deberá mediarle para sugerirle pistas que le permitan hacerlo- es porque busca causar "situaciones frustrantes para buscar cambios significativos" (Tébar, 2007, p.193) y para que autorregulen su cognición y emocionalidad gracias a la motivación y perseverancia.

En síntesis, un/a estudiante que ha desarrollado sus FC tiene mayor posibilidad de autorregularse puesto que conoce su propio proceso cognitivo: explora los datos, los relaciona significativamente y puede explicitarlos de manera oral o escrita en forma clara y precisa. Esta perspectiva no sólo incluye los factores cognitivos, sino también los afectivos motivacionales y los contextuales sociales (Pintrich, 2004). "Un estudiante apasionado por el conocimiento, tiene claro los objetivos de aprendizaje, sabe para dónde va, y hacia allí enfoca sus esfuerzos; establece procedimientos que le garanticen la aplicación de esos conocimientos y genera sus propios mecanismos para autorregular y controlar lo que aprende" (Urbina, 2016, p.26).

\section{Método}

Estudio exploratorio y descriptivo que busca determinar la autopercepción de las FC que tienen los/as estudiantes ingresados/as a las carreras renovadas de la ULS, en concordancia con el objetivo del proyecto de renovación de las carreras: "Potenciar en los estudiantes de carreras con currículos innovados, la capacidad de construir aprendizajes profundos y 
desarrollar habilidades afectivo motivacionales, a través de la utilización de estrategias de análisis de su proceso formativo".

\subsection{Muestra}

La población del estudio son todos los y las estudiantes que ingresaron el año 2015 y 2016 a las carreras que tienen un currículo renovados: Auditoría, Derecho, Enfermería, Ingeniería en Administración de Empresas, Ingeniería Comercial, Kinesiología, Licenciatura en Astronomía y Odontología. La muestra fue intencionada y se constituyó por seis estudiantes de cada una de las nueve carreras mencionadas. Se estableció como criterio de selección a aquellos estudiantes que cumplieran con las siguientes características:

a) Puntajes ponderados más altos en la Prueba de Selección Universitaria (PSU).

b) Puntajes más bajos en la PSU en las respectivas carreras.

c) Los más y los menos motivados por la carrera. Estos resultados se obtuvieron de la aplicación del instrumento diagnóstico que se realizó al ingreso a la institución.

La muestra final quedó constituida por un total de 112 estudiantes, de los cuales 54 ingresaron el año 2015 y 58 el 2016. El $62.9 \%$ corresponde a mujeres y el $37.1 \%$ hombres.

El rango de edades de los y las estudiantes para ambos años de ingreso osciló entre los 17 a los 28 años, primando el grupo etario de 17 a 20 años de edad, lo que equivale a un $81 \%$ de la muestra, distribuidos en un $46.7 \%$ de estudiantes de 17 y 18 años y un $34.3 \%$ de 19 y 20 años de edad.

Respecto a la elección de la carrera de los y las estudiantes que ingresaron el año 2015, 31 estudiantes de 54, postularon en primera opción a la carrera que estan cursando, lo que corresponde a un 69\%; 9 postularon a la carrera en segunda opción, lo que corresponde al 20\%; y 5 en tercera opción, lo que equivale al 11\%. El año 2016, de los 58 estudiantes de la muestra, 41 eligieron la carrera en primera opción, lo que equivale al $70.68 \%$, 15 en

8 Se entiende por currículo renovado: los planes de estudio cuyo perfil de egreso está planteado en competencias, centrado en el estudiante, cuyo foco es el aprendizaje. Se utilizan metodologías activo participantes y el número de horas de las asignatura está expresado en Sistema de Créditos Académicos Transferibles. 
segunda opción, lo que equivale al $25.86 \%$, y 2 en tercera y cuarta opción, lo que corresponde a un $1.71 \%$.

\subsection{Instrumentos}

Se aplicó la pauta de autopercepción de las FC extraída del texto "Metodología de la Mediación en el Programa de Enriquecimiento Instrumental (PEI)” (Martínez, 1991) la que considera las tres fases del acto mental propuestas por Feuerstein (1983, 1991). El instrumento está compuesto por 42 ítemes, el que se organiza en un escala tipo Likert con cinco opciones de respuesta: Siempre, Generalmente, A veces, Rara vez, y Nunca. Las aseveraciones son sustantivas, cuyas respuestas entregan orientación sobre las FC que los estudiantes creen tener más o menos desarrolladas.

Las FC fueron medidas en las tres fases del acto mental. La fase de entrada está constituida por ocho sub escalas, desde los ítems 1 al 14. Hay funciones que se valoran con un ítem, otras hasta con tres ítems, dependiendo de la pertinencia de cada ítem. La fase de elaboración está integrada por diez sub escalas, desde los ítems 15 al 31. De igual forma, hay funciones que se evalúan con un item y otras con cinco items. La fase de salida del acto mental se evalúa mediante ocho sub escalas, desde los items 32 al 42, de igual forma que las fases anteriores, algunas funciones se miden con un ítem y otras con tres ítems.

El proceso de validación y confiabilidad del instrumento comprendió diversas etapas: a) validación de contenido por revisión de expertos (Face Validity) (Escobar y Cuervo, 2008); b) medición de la confiabilidad por medio del Alfa de Cronbach (Cervantes, 2005); c) comprobación de la validez de constructo del modelo teórico por medio del Análisis Factorial Exploratorio.

Se utilizó el software SPSS versión 21 y el análisis factorial en las dimensiones de la escala (Entrada, Elaboración y Salida) y el Programa Factor versión 9.2 A. para calcular el análisis factorial.

La siguiente tabla muestra la confiabilidad del instrumento considerando los dos años de aplicación. 
Tabla 2 Confiabilidad escala de autopercepción de las funciones cognitivas de estudiantes. Ingreso años 2015

$$
\text { y } 2016 .
$$

\begin{tabular}{|l|l|l|l|l|}
\hline & \multicolumn{4}{|l|}{ Fases } \\
\hline & Fase Entrada & Elaboración & Fase Salida & Todos \\
\hline Ítems & $1-14$ & $15-31$ & $32-42$ & $1-42$ \\
\hline Alfa Cronbach & 0.85 & 0.89 & 0.89 & 0.95 \\
\hline
\end{tabular}

Fuente: Elaboración propia.

Como se observa en la tabla 2, la confiabilidad del instrumento es significativa para cada una de las fases y del instrumento en general. Para ello, se consideraron todos/as los y las estudiantes que participaron en el estudio y que ingresaron a las carreras de pregrado los años 2015 y 2016 (112 en total). En este sentido. el Alpha de Cronbach para todos los casos es mayor a .7, lo que demuestra una alta confiabilidad del instrumento independiente del año de ingreso de los y las estudiantes. A continuación se presenta la confiabilidad del instrumento por cada año en el cual se aplicó.

Tabla 3. Confiabilidad escala de autopercepción de las funciones cognitivas de estudiantes. Ingreso año 2015.

Fases

\begin{tabular}{|l|l|l|l|l|}
\hline & Fase Entrada & Elaboración & Fase Salida & Todos \\
\hline Ítems & $1-14$ & $15-31$ & $32-42$ & $1-42$ \\
\hline Alfa Cronbach & 0.86 & 0.90 & 0.90 & 0.96 \\
\hline
\end{tabular}

Fuente: Elaboración propia.

La tabla muestra altos índices de confiabilidad tanto en la tabla global como en cada una de sus fases en lo particular. Se consideró a los 54 estudiantes que ingresaron en el año 2015 y que participaron en el estudio.

Tabla 4 Confiabilidad escala de autopercepción de las funciones cognitivas de estudiantes. Ingreso año 2016.

Fases

\begin{tabular}{|l|l|l|l|l|}
\hline & Fase Entrada & Elaboración & Fase Salida & Todos \\
\hline Ítems & $1-14$ & $15-31$ & $32-42$ & $1-42$ \\
\hline Alfa Cronbach & 0.82 & 0.88 & 0.87 & 0.94 \\
\hline
\end{tabular}

Fuente: Elaboración propia. 
Al igual que en la tabla anterior, se observa un alto índice de confiabilidad desde una mirada global como en cada una de las fases en lo particular. De igual forma, se consideró a los 58 estudiantes que ingresaron el año 2016.

En resumen, la confiabilidad del instrumento original fue de .954 (95.4\%). Así mismo, para cada una de las dimensiones, las medidas del coeficiente Alfa de Cronbach obtenidos fueron superiores al $85 \%$ en las 3 subescalas. El cálculo de la confiabilidad por medio del Alfa de Cronbach, en todos los casos, mostró resultados superiores a 0.7, lo que indica que el instrumento y sus subescalas tienen una alta confiabilidad.

\subsection{Validez de constructo del instrumento}

Respecto al análisis factorial conviene aclarar que la escala usada en el instrumento es de tipo Likert, es decir, variables ordinales; en las cuales no podemos realizar el cálculo para las correlaciones de Pearson, las que se calculan en variables cuantitativas continuas. Por ello, siguiendo a Lorenzo y Ferrando, (2013) se calculó los estadísticos de la Matriz de Correlaciones Policóricas de los ítems de las subescalas de la Pauta, usando el software Factor propuesto por Lorenzo y Ferrando, (2006).

Una vez obtenida la Matriz de Correlaciones Policóricas, se utilizó el programa SPSS versión 21 para los cálculos siguientes: El test de adecuación de la muestra de KaiserMeyer Olkin fue de 0.905 y la prueba de esfericidad resultó significativa con una probabilidad del $95 \%(\mathrm{p}<.000)$, lo que permitió realizar un análisis factorial pertinente. Se recogieron saturaciones superiores a .3 y siguiendo el criterio de valores propios superiores a uno. Los resultados iniciales previos a la rotación, identificaron 3 factores, que resumían el $49.87 \%$ de la variabilidad total de los datos. Para confirmar la hipótesis de tridimensionalidad de la escala planteada en la teoría y para buscar el mejor ajuste, se decidió someter los resultados del Análisis de Componentes Principales con una rotación Varimax. Los resultados señalados corroboran que el instrumento tiene una estructura de 3 factores tal como se señala desde el punto de vista teórico.

\subsection{Procedimiento}


Previo a la aplicación de los instrumentos, se tomó contacto con los y las estudiantes y se les invitó a la Unidad de Mejoramiento Docente (UMD), dependiente de la Dirección de Docencia de la ULS. Se les dió a conocer el alcance del proyecto, la importancia de su participación y se les consultó si deseaban participar. Quienes aceptaron firmaron un consentimiento informado.

Los instrumentos se aplicaron a cada estudiante en una tablet, para que fuera más motivador, y las respuestas se almacenaron en el Google Drive, lo que facilitó la tabulación y el análisis posterior de los datos.

El análisis de los datos se realizó por cada uno de los y las estudiantes y a la vez por carrera. Los resultados se entregaron en forma personalizada, en formato verbal y escrito a cada estudiante. Para ello, se les citó a una entrevista en la UMD, se dialogó con ellos sobre sus resultados académicos y se profundizó sobre algunos aspectos específicos de las FC (considerando las tres fases del acto mental) y cómo fortalecerlas. Todos/as se sintieron identificados con las FC que aparecían más y menos desarrolladas. Se les dio sugerencias para fortalecer las menos avanzadas y algunas estrategias de estudio de acuerdo a la necesidad de cada estudiante.

Con los y las estudiantes que presentaron bajo desarrollo de las FC y un rendimiento académico insuficiente, se estableció la estrategia de acompañamiento basado en la teoría de la Experiencia de Aprendizaje Mediado y Modificabilidad Cognitiva de Reuven Feuerstein (1991) quien propone, entre otras, mediar las dificultades para aprender a través de la intencionalidad-reciprocidad, significado y trascendencia. Para ello se trabajó con los y las estudiantes en el fortalecimiento de las FC de cada fase del acto mental, realizando variadas actividades específicas tales como focalizar la atención, establecer metas alcanzables, explicitar criterios para la selección y organización de los datos, habilidad en la búsqueda de información en forma organizada y en diversas fuentes, incrementar el vocabulario, establecer diferentes códigos para incrementar las habilidades lingüísticas, con el propósito de optimizar los aprendizajes.

Simultáneamente, a los/as directores/as de las carreras y a los/as profesores/as que dictan las asignaturas que cursaban los y las estudiantes, se les entregó un informe con los 
resultados de las principales fortalezas y aspectos por mejorar y se les sugirieron estrategias para fortalecer la modificabilidad cognitiva y con ello, mejorar los aprendizajes en las respectivas carreras.

\section{Resultados}

Los hallazgos revelan que las tres fases del acto mental están íntimamente asociadas, observándose claramente que hay deficiencias en las FC de la fase de entrada y en la de elaboración y dificultades en la fase de salida. Por ejemplo, si en la fase de entrada de un/a estudiante de Odontología hay deficiencia en la constancia y permanencia del objeto, será más difícil que establezca relaciones en una comparación en la fase de elaboracin, aspecto crucial para la precisión y exactitud en la fase de salida. De la misma manera, si en la fase de entrada de un/a estudiante de la carrera de Enfermería hay deficiencia en la FC de precisión y exactitud es muy probable que se le dificulte la planificación de la conducta en la fase de elaboración, con un resultado impreciso en la fase de salida. Así podemos analizar cada uno de los resultados y, además, inferir las dificultades que tendrían los futuros profesionales en el ejercicio de su profesión .

Tabla 5. Funciones cognitivas con menor desarrollo en las tres fases del acto mental, según la autopercepcion de los y las estudiantes que ingresaron a las carreras renovadas el año 2015.

\begin{tabular}{|c|c|c|c|}
\hline Carreras & Fase de entrada & Fase de elaboración & Fase de salida \\
\hline Odontología & $\begin{array}{l}\text { Percepción. } \\
\text { Precisión y exactitud. } \\
\text { Constancia y } \\
\text { permanencia del objeto. }\end{array}$ & $\begin{array}{l}\text { Amplitud campo mental. } \\
\text { Conducta comparativa. }\end{array}$ & Control de la respuesta. \\
\hline Enfermería & $\begin{array}{l}\text { Percepción. } \\
\text { Precisión y exactitud. } \\
\text { Constancia y } \\
\text { permanencia del objeto. }\end{array}$ & $\begin{array}{l}\text { Percepción holística de } \\
\text { la realidad. } \\
\text { Planificación de la } \\
\text { conducta. }\end{array}$ & $\begin{array}{l}\text { Elaboración y } \\
\text { desinhibición en la } \\
\text { comunicación. } \\
\text { Transporte visual. }\end{array}$ \\
\hline Kinesiologia & $\begin{array}{l}\text { Percepción. } \\
\text { Exploración sistemática. } \\
\text { Precisión y exactitud. } \\
\text { Habilidades lingüísticas. }\end{array}$ & $\begin{array}{l}\text { Percepción y definición } \\
\text { del problema. } \\
\text { Planificación de la } \\
\text { conducta. } \\
\text { Amplitud del campo } \\
\text { mental. }\end{array}$ & $\begin{array}{l}\text { Elaboración y } \\
\text { desinhibición en la } \\
\text { comunicación. } \\
\text { Precisión y exactitud. } \\
\text { Transporte visual. }\end{array}$ \\
\hline $\begin{array}{l}\text { Ingeniería en } \\
\text { Administración de } \\
\text { empresas }\end{array}$ & $\begin{array}{l}\text { Exploración sistemática. } \\
\text { Precisión y exactitud. } \\
\text { Habilidades lingüísticas. } \\
\text { Constancia y } \\
\text { permanencia. } \\
\text { Atención a dos o más }\end{array}$ & $\begin{array}{l}\text { Percepción y definición } \\
\text { del problema. } \\
\text { Conducta comparativa. } \\
\text { Pensamiento hipotético. } \\
\text { Amplitud del campo } \\
\text { mental. }\end{array}$ & $\begin{array}{l}\text { Precisión y exactitud. } \\
\text { Transporte visual. }\end{array}$ \\
\hline
\end{tabular}




\begin{tabular}{|c|c|c|c|}
\hline & fuentes de información. & & \\
\hline Ingeniería Comercial & $\begin{array}{l}\text { Precisión y exactitud. } \\
\text { Habilidades lingüísticas. } \\
\text { Atención a dos o más } \\
\text { fuentes de información. }\end{array}$ & $\begin{array}{l}\text { Planificación de la } \\
\text { conducta. } \\
\text { Amplitud del campo } \\
\text { mental. } \\
\text { Conducta comparativa. }\end{array}$ & $\begin{array}{l}\text { Elaboración y } \\
\text { desinhibición de la } \\
\text { comunicación. } \\
\text { Comunicación explícita. } \\
\text { Habilidades lingüísticas. }\end{array}$ \\
\hline Auditoría & $\begin{array}{l}\text { Precisión y exactitud. } \\
\text { Constancia y } \\
\text { permanencia del objeto. } \\
\text { Atención a dos o más } \\
\text { fuentes de información. }\end{array}$ & $\begin{array}{l}\text { Percepción y definición } \\
\text { del problema. } \\
\text { Conducta comparativa. } \\
\text { Planificación de la } \\
\text { conducta. } \\
\text { Amplitud del campo } \\
\text { mental. } \\
\text { Clasificación cognitiva. }\end{array}$ & $\begin{array}{l}\text { Comunicación explícita. } \\
\text { Precisión y exactitud. }\end{array}$ \\
\hline Derecho & $\begin{array}{l}\text { Precisión y exactitud. } \\
\text { Constancia y } \\
\text { permanencia del objeto. }\end{array}$ & $\begin{array}{l}\text { Percepción y definición } \\
\text { del problema. } \\
\text { Planificación de la } \\
\text { conducta. } \\
\text { Amplitud del campo } \\
\text { mental. } \\
\text { Clasificación cognitiva. }\end{array}$ & $\begin{array}{l}\text { Comunicación explícita. } \\
\text { Transporte visual. }\end{array}$ \\
\hline Ingeniería en alimentos & $\begin{array}{l}\text { Percepción. } \\
\text { Habilidades lingüísticas. } \\
\text { Constancia y } \\
\text { permanencia del objeto. }\end{array}$ & $\begin{array}{l}\text { Pensamiento hipotético. } \\
\text { Planificación de la } \\
\text { conducta. } \\
\text { Amplitud del campo } \\
\text { mental. } \\
\text { Clasificación cognitiva. }\end{array}$ & $\begin{array}{l}\text { Comunicación explícita. } \\
\text { Control de la respuesta. } \\
\text { Transporte visual. }\end{array}$ \\
\hline $\begin{array}{l}\text { Licenciatura en } \\
\text { Astronomía }\end{array}$ & $\begin{array}{l}\text { Percepción. } \\
\text { Precisión y exactitud. } \\
\text { Atención a dos o más } \\
\text { fuentes de información. }\end{array}$ & $\begin{array}{l}\text { Planificación de la } \\
\text { conducta. } \\
\text { Amplitud del campo } \\
\text { mental. }\end{array}$ & $\begin{array}{l}\text { Habilidades lingüísticas. } \\
\text { Transporte visual. }\end{array}$ \\
\hline
\end{tabular}

Fuente: Elaboración propia

En la fase de entrada de la información se observa menor desarrollo en la FC Precisión y exactitud en 8 de 9 carreras. Le siguen Constancia y permanencia del objeto (6 de 9), Percepción clara (5 de 9), Habilidades lingüísticas (4 de 9), Atención a dos o más fuentes de información (4 de 9) y Exploración sistemática (2 de 9).

En la fase de elaboración de la información se observa menor desarrollo en la FC Amplitud del campo mental en 8 de 9 carreras. Le siguen Planificación de la conducta ( 7 de 9), Conducta comparativa (4 de 9), Percepción y definición del problema (4 de 9), Clasificación cognitiva (3 de 9), Pensamiento hipotético (2 de 9) y Percepción holística de la realidad (1 de 9). 
En la fase de salida de la información se observa menor desarrollo en la FC Transporte visual en 6 de 9 carreras. Le siguen Comunicación explícita (4 de 9), Elaboración y desinhibición de la comunicación (3 de 9), Precisión y exactitud (3 de 9), Habilidades lingüísticas (2 de 9), y Control de la respuesta (2 de 9).

Es fácil deducir que si los y las estudiantes tienen un menor desarrollo de la FC Precisión y exactitud en la Fase de Entrada, los datos que recojan serán dispersos, lo que dificultará la posterior elaboración. Si en la fase de Elaboración la FC menos desarrollada es la Amplitud del campo mental, hay mayores probabilidades de pensar de manera egocéntrica, y si en la Fase de salida, la FC menos desarrollada es el Transporte visual, nos encontramos con estudiantes a los que se les dificultará trasladar los datos a distintos contextos.

Tabla 6. Funciones cognitivas con menor desarrollo en las tres fases del acto mental, según la autopercepcion de los y las estudiantes que ingresaron a las carreras renovadas el año 2016.

\begin{tabular}{|l|l|l|l|}
\hline \multicolumn{1}{|c|}{ Carreras } & \multicolumn{1}{|c|}{ Fase de entrada } & \multicolumn{1}{|c|}{ Fases de elaboración } & \multicolumn{1}{c|}{ Fase de salida } \\
\hline Odontología & $\begin{array}{l}\text { Exploración } \\
\text { sistemática. } \\
\text { Orientación espacial. }\end{array}$ & Amplitud del campo mental. & $\begin{array}{l}\text { Comunicación explícita. } \\
\text { Elaboración y } \\
\text { desinhibición en la } \\
\text { comunicación. }\end{array}$ \\
\hline Enfermería & $\begin{array}{l}\text { Percepción. } \\
\text { Atención a dos o más } \\
\text { fuentes de } \\
\text { información. }\end{array}$ & $\begin{array}{l}\text { Pensamiento hipotético. } \\
\text { Amplitud del campo mental. } \\
\text { Conducta interiorizada. }\end{array}$ & Comunicación explícita. \\
\hline Kinesiologia & $\begin{array}{l}\text { Percepción. } \\
\text { Precisión y exactitud. }\end{array}$ & $\begin{array}{l}\text { Conducta comparativa. } \\
\text { Evidencia lógica. } \\
\text { Pensamiento hipotético. } \\
\text { Planificación de la conducta. } \\
\text { Amplitud del campo mental. } \\
\text { Conducta interiorizada. } \\
\text { Clasificación cognitiva. }\end{array}$ & $\begin{array}{l}\text { Elaboración y } \\
\text { desinhibición en la } \\
\text { comunicación. } \\
\text { Precisión y exactitud. } \\
\text { Transporte visual. }\end{array}$ \\
\hline $\begin{array}{l}\text { Ingeniería en } \\
\text { Administración de } \\
\text { empresas }\end{array}$ & $\begin{array}{l}\text { Atención a dos o más } \\
\text { fuentes de } \\
\text { información. }\end{array}$ & $\begin{array}{l}\text { Planificación de la conducta. } \\
\text { Amplitud del campo mental. }\end{array}$ & $\begin{array}{l}\text { Elaboración y } \\
\text { desinhibición en la } \\
\text { comunicación. } \\
\text { Control de la respuesta. }\end{array}$ \\
\hline $\begin{array}{l}\text { Ingeniería } \\
\text { Comercial }\end{array}$ & $\begin{array}{l}\text { Percepción. } \\
\text { Habilidades } \\
\text { lingüísticas. }\end{array}$ & $\begin{array}{l}\text { Percepción y definición del } \\
\text { problema. } \\
\text { Evidencia lógica. } \\
\text { Planificación de la conducta. } \\
\text { Amplitud del campo mental. } \\
\text { Conducta interiorizada. } \\
\text { Clasificación cognitiva. }\end{array}$ & $\begin{array}{l}\text { Habilidades lingüísticas. } \\
\text { Elaboracion y } \\
\text { desinhibicion en la } \\
\text { comunicación. } \\
\text { Precisión y exactitud. } \\
\text { Transporte visual. }\end{array}$ \\
\hline Auditoría & $\begin{array}{l}\text { Precisión y exactitud. } \\
\text { Constancia y }\end{array}$ & $\begin{array}{l}\text { Planificación de la conducta. } \\
\text { Amplitud del campo mental. }\end{array}$ & Transporte visual. \\
\hline
\end{tabular}




\begin{tabular}{|c|c|c|c|}
\hline & $\begin{array}{l}\text { permanencia. } \\
\text { Exploración } \\
\text { sistemática. }\end{array}$ & & \\
\hline Derecho & $\begin{array}{l}\text { Habilidades } \\
\text { lingüísticas. } \\
\text { Orientación espacial. }\end{array}$ & $\begin{array}{l}\text { Amplitud el campo mental. } \\
\text { Conducta interiorizada }\end{array}$ & $\begin{array}{l}\text { Elaboración y } \\
\text { desinhibición en la } \\
\text { comunicación } \\
\text { Comunicación explícita. } \\
\text { Transporte visual }\end{array}$ \\
\hline $\begin{array}{l}\text { Ingeniería en } \\
\text { alimentos }\end{array}$ & $\begin{array}{l}\text { Precisión y exactitud } \\
\text { Atención a dos o más } \\
\text { fuentes de } \\
\text { información. }\end{array}$ & $\begin{array}{l}\text { Planificación de la conducta. } \\
\text { Amplitud del campo mental. } \\
\text { Evidencia lógica. }\end{array}$ & Transporte visual. \\
\hline $\begin{array}{l}\text { Licenciatura en } \\
\text { Astronomía }\end{array}$ & $\begin{array}{l}\text { Precisión y exactitud. } \\
\text { Habilidades } \\
\text { lingüísticas. } \\
\text { Orientación temporal. } \\
\text { Constancia y } \\
\text { permanencia. } \\
\text { Atención a dos o más } \\
\text { fuentes de } \\
\text { información. }\end{array}$ & $\begin{array}{l}\text { Percepción holística de la } \\
\text { realidad. } \\
\text { Pensamiento hipotético. } \\
\text { Planificación de la conducta. } \\
\text { Amplitud del campo mental. } \\
\text { Conducta interiorizada. } \\
\text { Clasificación cognitiva. }\end{array}$ & $\begin{array}{l}\text { Elaboración y } \\
\text { desinhibición en la } \\
\text { comunicación. } \\
\text { Precisión y exactitud. } \\
\text { Transporte visual. }\end{array}$ \\
\hline
\end{tabular}

Fuente: Elaboración propia

En la fase de entrada de la información se observa menor desarrollo en la FC Atención a dos o más fuentes de información y en Precisión y exactitud, en 4 de 9 carreras. Le siguen Percepción (3 de 9), Habilidades lingüísticas, y Orientación espacial (3 de 9), Constancia y permanencia y Exploración sistemática (2 de 9).

En la fase de elaboración de la información se observa menor desarrollo en la FC Amplitud del campo mental en la totalidad de las carreras (9 de 9) carreras. Le siguen Planificación de la conducta (6 de 9), Conducta interiorizada (5 de 9), Pensamiento hipotético (3 de 9), Clasificación cognitiva y Evidencia lógica (3 de 9), Percepción y definición del problema y Percepción holística de la realidad (1 de 9).

En la fase de salida de la información se observa menor desarrollo en la FC Elaboración y desinhibición en la comunicación y Transporte visual en 6 de 9 carreras. Le siguen Comunicación explícita (4 de 9), Precisión y exactitud (3 de 9), Habilidades linguiísticas y Control de la respuesta (1 de 9).

Como en la tabla del año anterior, es fácil deducir que si los y las estudiantes tienen un menor desarrollo de la FC Atención a dos o más fuentes de información y en Precisión y 
exactitud en la Fase de Entrada, tendrán dificultades para fijarse en más de un detalle de los datos que recojan y además éstos pueden recojerse de manera dispersa. Si en la fase de Elaboración la FC menos desarrollada, en todas las carreras, es la Amplitud del campo mental hay mayores probabilidades de pensar de manera egocéntrica, y si en la Fase de salida, las FC menos desarrolladas son la Elaboración y desinhibición en la comunicación y el Transporte visual, nos encontramos con estudiantes a quienes se les dificultará entregar una respuesta clara y precisa y trasladar los datos a distintos contextos.

En los dos años de aplicación del instrumento se observa convergencia en las FC menos desarrolladas en cada una de las fases del acto mental.

Tabla 7. Resultados de las Funciones Cognitivas menos desarrolladas, según la percepción de los y las estudiantes que ingresaron a las carreras renovadas los años 2015 - 2016.

\begin{tabular}{|l|l|l|}
\hline \multicolumn{1}{|c|}{ Fases Acto Mental } & \multicolumn{1}{c|}{$\mathbf{2 0 1 5}$} & \multicolumn{1}{c|}{$\mathbf{2 0 1 6}$} \\
\hline Fase de Entrada & Precisión y exactitud (8 de 9) & $\begin{array}{l}\text { Atención a dos o más fuentes de } \\
\text { información y Precisión y } \\
\text { exactitud (4 de 9) }\end{array}$ \\
\hline Fase de Elaboración & $\begin{array}{l}\text { Amplitud del campo mental (8 de } \\
\text { 9) }\end{array}$ & $\begin{array}{l}\text { Amplitud del campo mental (9 de } \\
\text { 9) }\end{array}$ \\
\hline Fase de Salida & Transporte visual (6 de 9) & $\begin{array}{l}\text { Elaboración y desinhibición en la } \\
\text { comunicación y Transporte visual } \\
\text { (6 de 9) }\end{array}$ \\
\hline
\end{tabular}

Fuente Elaboracion propia

Como el propósito del estudio fue potenciar en los y las estudiantes la capacidad de construir aprendizajes profundos utilizando estrategias de análisis de su propio proceso formativo, nos interesó no solo indagar en sus dificultades, sino también conocer las FC más desarrolladas para fortalecer su desarrollo integral.

Tabla 8. Funciones cognitivas más desarrolladas, en general, según la autopercepcion de los y las estudiantes, que ingresaron a las carreras renovadas los años 2015 - 2016.

\begin{tabular}{|l|l|l|}
\hline \multicolumn{1}{|c|}{ Fase de Entrada } & \multicolumn{1}{c|}{ Fase de Elaboración } & \multicolumn{1}{c|}{ Fase de Salida } \\
\hline Orientación temporal & $\begin{array}{l}\text { Percepción de la } \\
\text { realidad }\end{array}$ & Habilidades lingüísticas \\
\hline $\begin{array}{l}\text { Atención a dos o más fuentes de } \\
\text { información. }\end{array}$ & Distinción de datos relevantes & Control de la respuesta \\
\hline
\end{tabular}

Fuente Elaboración propia

\section{Discusión}


La investigación nos permite señalar que:

- Las FC menos desarrolladas son similares en estudiantes de distintas áreas disciplinares, independientemente de lo que estudia y del año de ingreso a la universidad

- Los/as estudiantes desconocen su propia capacidad de autorregulación.

- Los/as estudiantes establecen un locus de control externo responsabilizando a otros.

- Todo estudiante puede modificarse cognitivamente (neuroplasticidad).

- Si hubiere resistencia del/a estudiante se puede superar gracias a EAM.

- La modificabilidad cognitiva es intencional y favorece la propensión a aprender.

- La EAM favorece la mejoría de las FC, la metacognición y la autorregulación.

- El que enseña es responsable por la atribución, expectativas y rendimiento de sus estudiantes, gracias a las EAM y la creación de ambientes activos modificantes

\section{Referencias bibliográficas}

Calvo, C. (2016). Del mapa escolar al territorio educativo. Disoñando la escuela desde la educación. La Serena: Editorial Unviersidad de La Serena

Cervantes, V. (2005). Interpretaciones del coeficiente alpha de cronbach. Avances en Medición, 3, 928.

Cuervo, A. (2008). Validez de contenido y juicio de expertos: una aproximación a su utilización. Avances en Medición, 6, 27-36.

Facse, E. (2007). Aprendizaje profundo y superficial. Educación y Ciencias de la Salud. 4 (1), 7-8.

Feuerstein, R. (1983). Instrumental Enrichment. Baltimore: University Park Press.

(1991). Mediated Learning Experience (MLE). Theoretical, Psichosocial and Learning Implications. London: Freund Publishing House ltd.

Ferguson, Marilyn. 1988. La Conspiracion de Acuario. Barcelona: Editorial Kairos. 
López de Maturana, S. (2010). Maestros en el Territorio. La Serena: Editorial Universidad de La Serena.

Lorenzo, U., Ferrando, P.J. (2006). FACTOR: A computer program to fit the exploratory factor analysis model. Behavioral Research Methods, Instruments and Computers, $38,(1), 88-91$.

- (2013). FACTOR 9.2 A Comprehensive Program for Fitting Exploratory and Semiconfirmatory Factor Analysis and IRT Models. Applied Psychological Measurement, 37 (6), 497-498.

Martínez, J. M. (1991). Metodología de la Mediación en el P.E.I. Madrid: Bruño.

Modelo Educativo (2011). Universidad de La Serena.

Morin, E. (2001). Los siete saberes necesarios para la educación del futuro. Barcelona:Paidós.

Prieto, M. (1989). Modificabilidad Cognitiva y P.E.I. Madrid: Bruño.

Pintrich, P. (2004). Un marco conceptual para evaluar la motivación y el aprendizaje autorregulado en estudiantes universitarios. Educational Psychology Review 16 (4), 385- 407. http://hdl.handle.net/2027.42/44454

Unidad de Mejoramiento Docente (2014) Fortalecimiento de la Unidad de Mejoramiento Docente de la Universidad de la Serena mediante la creación e implementación del área de Innovación y Transferencia en aprendizaje, docencia y gestión curricular: una mejora sistémica en el proceso de Innovación Curricular. Proyecto de Mejoramiento de Programas, del Ministerio de Educación, Chile.

Tébar, L. (2007). El profesor mediador del aprendizaje. Bogotá: Magisterio. 